\title{
SYMMETRIES AND RANK DEFICIENCY IN THE ORBIT DETERMINATION AROUND ANOTHER PLANET
}

\author{
CLAUDIO BONANNO AND ANDREA MILANI \\ Dipartimento di Matematica \\ Università di Pisa \\ via Buonarroti 2, 56100 Pisa (Italy) \\ email: bonanno@dm.unipi.it \\ milani@dm.unipi.it
}

\begin{abstract}
We study the possible degeneracies for the normal matrix of the observations from the Earth of the motion of a satellite around a planet, and give the possible solutions to the loss of precision in the orbit determination caused by the rank deficiency. Finally we discuss the methods available to control the instability in the orbit determination resulting from the degeneracy.
\end{abstract}

Keywords: orbit determinations, symmetries

\section{Introduction}

The problem of orbit determination of a satellite orbiting around another planet has been faced many times in the last 40 years, during many planetary orbiter missions. We consider the case of a planetary orbiter observed from a tracking station on the Earth by range and range-rate observations.

It has long been known (analytical studies and numerical simulations) that some orbital elements are much better determined than others in the case of planetary orbiter, due to geometrical symmetries in the system. Some results for an analytical expression of the value of the degeneracy obtained are shown in (Russell and Thurman, 1989). Also, the particular configurations of the orbit that can cause problems in determining some elements have been analyzed (Wood, 1986).

In this paper we study the presence of the geometrical symmetries from an analytical point of view, proving that some degeneracies in the determination of some parameters cannot be avoided. In particular, we show that in an ideal situation (no relative motion between the Earth and the planet being orbited, and perfectly spherically symmetric planet), one of the parameters cannot be determined at all. But, if we consider the realistic situation, we find that the relative motion between the Earth and the planet and the oblateness of the planet cause only a small perturbation on the symmetry we found in the ideal case. Hence we still have that one of the parameters is much worse determined than the others; we give an estimate on the loss of precision resulting from the approximated degeneration. Finally we 
discuss the methods available to control the instability in the orbit determination resulting from the degeneracy.

\section{The problem}

We consider the problem of the orbit determination for a satellite orbiting a planet, different from the Earth, using the two body approximation for the planet-satellite system. We suppose that there is made a tracking of the satellite's orbit from the Earth, and the observations made are the satellite's geocentric distance and its geocentric radial velocity. Then, if we call $\vec{r}$ the planet-satellite vector and $\vec{R}$ the Earth-planet vector, the observations are the range and the range-rate, that is $\|\vec{r}+\vec{R}\|$ and $\frac{d}{d t}\|\vec{r}+\vec{R}\|$. We are assuming that the position of the observer can be identified with the center of the Earth.

In this system there are six parameters, that are the six orbital elements of the satellite's orbit. Let $\mathcal{P}$ be the parameters space and $O$ the observations space. Then $\operatorname{dim} P=6$ and $\operatorname{dim} O=2 N$, where $N$ is the number of observations made, and the factor 2 comes from the fact that each observation corresponds to two different quantities. So, there is a real function

$$
F: \mathcal{P} \rightarrow O
$$

that represents the observations.

From the observations function it is possible in principle to obtain the six parameters that specify the orbit of the satellite. One of the used methods is the least squares method (Gauss, 1809). This method, beside the nominal solution, provides the so-called confidence ellipsoid, that is an ellipsoid in the parameters space of possible solutions. This ellipsoid, which has the center on the nominal solution, is a level hypersurface of a probability distribution for the solution of the problem. Given the Jacobian $B$ of the observations function, that is

$$
B=\left(\frac{\partial F}{\partial \vec{p}}\right)
$$

we define the normal matrix $C$ by

$$
C=B^{T} B
$$

The confidence ellipsoid $E$ is then defined by

$$
E=\left\{\vec{p}^{t} C \vec{p}=\sigma^{2}\right\}
$$

for a given parameter $\sigma$. The axes of the ellipsoid $E$ are given by the inverse of the eigenvalues of the normal matrix $C$. Hence if $C$ had $k$ null eigenvalues, then the ellipsoid $E$ would be degenerate in $k$ dimensions. In this case the normal matrix $C$ has a rank deficiency. 
Definition 2.1. The order of the rank deficiency is the number of null eigenvalues of the normal matrix.

The problem of degeneration of the confidence ellipsoid, related to the determination of the parameters of the orbit, is associated to the presence of symmetries for the problem.

Definition 2.2. Given the function $F: \mathcal{P} \rightarrow O$ of the observations, there is an exact symmetry if there exists a Lie group $G$ of transformations of the space $P$ into itself, such that

$$
\forall g \in G \text { and } \vec{p} \in \mathcal{P} \quad F(g \vec{p})=F(\vec{p}),
$$

where $g \vec{p}$ denotes the action of $G$ on $\mathcal{P}$ (Milani and Melchioni, 1989). The group $G$ is called a group of symmetries. We call local group of symmetries a neighbourhood of the identity of a group such that each of its elements is a symmetry, that is equation (2) holds.

Theorem 2.3. The order of the rank deficiency is equal to the dimension of the largest local group of symmetries $G$, whose action is effective (no subgroup acts trivially).

Proof. Let $\vec{p}$ be a point in the parameter space $\mathcal{P}$, and consider the orbit of $\vec{p}$ by the effective action of a Lie group $G$ of dimension $k$. From the definition of effective action, it follows that the orbit of $\vec{p}$ is a sub-manifold of $\mathcal{P}$ of dimension $k$. Then it is possible to define a local coordinate system for the space $\mathcal{P}$, in which the first $k$ vectors $e_{1}, \ldots, e_{k}$ parameterize the orbit of $\vec{p}$.

Let's assume that $G$ is the largest local group of symmetries for the observations function $F$. Then $F$ must be independent on the first $k$ components $e_{1}, \ldots, e_{k}$ of the space $\mathcal{P}$. Looking now at the form of the normal matrix $C$, defined above, obtained from the function $F$ at $\vec{p}$, we have that it has at least $k$ null eigenvalues, hence a rank deficiency of order $\geq k$.

Moreover, if the normal matrix $C$ had a rank deficiency of order $n>k$, then there would be a local coordinates system such that the observations function $F$ does not depend on the first $n$ components. Then the translations on the first $n$ coordinates define a Lie algebra $g$ of dimension $n$ and, using the exponential map, we obtain a group $G^{\prime}$, such that a neighbourhood of the identity is a local group of symmetries with effective action. But the dimension of $G^{\prime}$ is equal to the dimension of the Lie algebra, contradicting the hypothesis that $G$ is the largest local group of symmetries.

Hence if there is a group of symmetries $G$ for our problem, then the associated normal matrix $C$ has a rank deficiency of order equal to the dimension of the group. This order is equal to the number of degenerate dimensions in the confidence ellipsoid, and hence is equal to the number of parameters that cannot be determined by 
the observations of the satellite. We show in the next section that for our problem there is a one dimensional group of symmetries, hence we cannot determine one parameter.

\section{The exact symmetry}

Let's suppose that the planet is perfectly spherically symmetric and that there is no relative motion between the Earth and the planet. In this simplified case, we find an exact one-dimensional symmetry for the problem.

To study our system, consider first the case in which we have just one observation, that is $N=1$. Then $\operatorname{dim} O=2$, and let $F^{1}$ and $F^{2}$ be the two components of the observations function: $F^{1}(\vec{p})=\|\vec{r}+\vec{R}\|$ and $F^{2}(\vec{p})=\frac{d}{d t}\|\vec{r}+\vec{R}\|$, where $\vec{p} \in \mathcal{P}$ is the vector of the orbital elements of the satellite to be determined. The first component $F^{1}$ determines a sphere $\Sigma$ of radius $\|\vec{r}+\vec{R}\|$ and center on the center of the Earth. The second component $F^{2}$ gives the geocentric radial velocity of the satellite, that is the component of the geocentric velocity orthogonal to the sphere $\Sigma$.

We can parameterize the parameter space $\mathcal{P}$ as $\mathbb{R}^{3} \times \mathbb{R}^{3}$, where $\vec{p}=\left(\vec{r}, \frac{d}{d t} \vec{r}\right)$ (i.e. instead of considering the six keplerian orbital elements, we consider the radius vector and the velocity). Then, in this case, $N=1$, there is a four dimensional manifold of initial conditions corresponding to the same observations (the product of two sphere). Thus there is a four dimensional local group of symmetries operating effectively on this manifold.

On the contrary, for $N \geq 3$ there is (generically) only a one dimensional group of symmetries for our problem. The symmetries correspond to the rotation of all the system planet-satellite around the Earth-planet vector $\vec{R}$.

\subsection{The mathematical formulation of the symmetry}

Let's change our system of coordinates on the parameters space $\mathcal{P}$. Let $x y z$ be a cartesian reference frame with center on the center of the Earth, with the $y$ axis in the direction of the planet, the $x$ axis orthogonal to the $y$ axis and in some reference plane, and the $z$ axis orthogonal to the plane. Let $r$ and $v$ be the length of the planet-satellite vector and of the satellite's velocity with respect to the planet, respectively. Let $\vartheta_{r}$ be the angle between $\vec{r}$ and the $y$ axis, and $\varphi_{r}$ the angle between the projection of $\vec{r}$ on the $x z$ plane and the $x$ axis (see Figure 1). Analogously we can define for the velocity vector the angles $\vartheta_{v}$ and $\varphi_{v}$ as for $\vec{r}$. The coordinates transformations are given by

$$
\left\{\begin{array} { l } 
{ r _ { x } = r \operatorname { s i n } \vartheta _ { r } \operatorname { c o s } \varphi _ { r } } \\
{ r _ { y } = r \operatorname { c o s } \vartheta _ { r } } \\
{ r _ { z } = r \operatorname { s i n } \vartheta _ { r } \operatorname { s i n } \varphi _ { r } }
\end{array} \quad \left\{\begin{array}{l}
v_{x}=v \sin \vartheta_{v} \cos \varphi_{v} \\
v_{y}=v \cos \vartheta_{v} \\
v_{z}=v \sin \vartheta_{v} \sin \varphi_{v}
\end{array}\right.\right.
$$

In the following it will be useful to consider a further change of coordinates, namely $\alpha=\varphi_{r}-\varphi_{v}$ and $\beta=\varphi_{r}+\varphi_{v}$. 


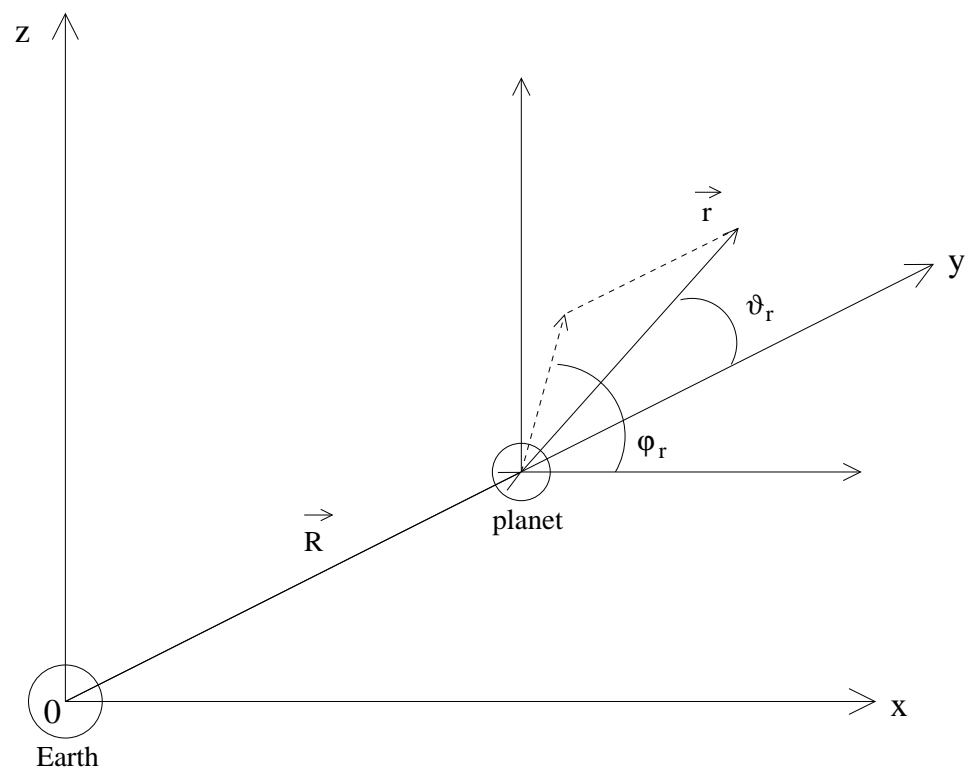

Figure 1. The geocentric reference frame with the new spherical coordinates for the satellite

Let's consider the group $S^{1}=\{\psi \in \mathbb{R}(\bmod 2 \pi)\}$ acting on $\mathcal{P}$ by

$$
g_{\psi}(\vec{p})=g_{\psi}\left(r, \vartheta_{r}, \varphi_{r}, v, \vartheta_{v}, \varphi_{v}\right)=\left(r, \vartheta_{r}, \varphi_{r}+\psi, \nu, \vartheta_{v}, \varphi_{v}+\psi\right) \forall \vec{p} \in \mathcal{P}
$$

that corresponds to a rotation of the parameters of an angle $\psi$ around the Earthplanet vector $\vec{R}$. We remark that the action of $S^{1}$ affects only $\beta$, leaving unchanged $\left(r, v, \vartheta_{r}, \vartheta_{v}, \alpha\right)$.

Let's suppose to have $N+1$ observations, and call $F_{j}^{i}$, for $i=1,2$ and $j=$ $0, \ldots, N$ the components of the observations function $F: \mathcal{P} \rightarrow O$. We then have $F_{j}^{1}=\left\|\vec{r}\left(t_{j}\right)+\vec{R}\left(t_{j}\right)\right\|$ and $F_{j}^{2}=\frac{d}{d t}\left\|\vec{r}\left(t_{j}\right)+\vec{R}\left(t_{j}\right)\right\|$, where $t_{j}, j=0, \ldots, N$, are the times of the observations. We consider the time $t_{0}$ as the reference epoch, that is we suppose that the six parameters to be determined are $r\left(t_{0}\right), v\left(t_{0}\right), \vartheta_{r}\left(t_{0}\right), \vartheta_{v}\left(t_{0}\right), \alpha\left(t_{0}\right)$ and $\beta\left(t_{0}\right)$, and they represent the initial conditions of the satellite's orbit. Obviously all the other quantities at times $t_{j}$ with $j \neq 0$ are functions of our initial conditions.

Theorem 3.1. If there is no relative motion between the Earth and the planet, and the planet is spherically symmetric, then $\frac{\partial}{\partial \beta\left(t_{0}\right)} F_{j}^{i}=0$ for each $i=1,2$ and $j=$ $0, \ldots, N$. Hence the group of rotations $S^{1}$ defined as above gives an exact symmetry.

Proof. Let's write the components of the observations function $F$ in the given coordinates. Using the fact that there is no relative motion between the Earth and 
the planet, we have that $\vec{R}\left(t_{j}\right)=\vec{R}$ for each $j=0, \ldots, N$. So, we get

$$
\left\{\begin{aligned}
F_{j}^{1}= & \left(r^{2}\left(t_{j}\right)+R^{2}+2 r\left(t_{j}\right) R \cos \left(\vartheta_{r}\left(t_{j}\right)\right)\right)^{\frac{1}{2}} \\
F_{j}^{1} F_{j}^{2}= & r\left(t_{j}\right) v\left(t_{j}\right)\left[\sin \left(\vartheta_{r}\left(t_{j}\right)\right) \sin \left(\vartheta_{v}\left(t_{j}\right)\right) \cos \left(\alpha\left(t_{j}\right)\right)+\right. \\
& \left.\cos \left(\vartheta_{r}\left(t_{j}\right)\right) \cos \left(\vartheta_{v}\left(t_{j}\right)\right)\right]+v\left(t_{j}\right) R \cos \left(\vartheta_{v}\left(t_{j}\right)\right)
\end{aligned}\right.
$$

We need to have the observations functions $F_{j}^{i}$, with $j \geq 1$, in terms of the initial conditions $r\left(t_{0}\right), v\left(t_{0}\right), \vartheta_{r}\left(t_{0}\right), \vartheta_{v}\left(t_{0}\right), \alpha\left(t_{0}\right), \beta\left(t_{0}\right)$, that are the parameters of the orbit to be determined. Looking at classical methods of orbit determination, we find that a useful tool are the $f$ and $g$ series (Escobal, 1965). That is we can write $\vec{r}\left(t_{j}\right), \vec{v}\left(t_{j}\right)$, $j=1, \ldots, N$, as functions of $\vec{r}\left(t_{0}\right), \vec{v}\left(t_{0}\right)$, using the fact that they are solutions to the same two body problem. We have that for $j=1, \ldots, N$

$$
\left\{\begin{array}{l}
\vec{r}\left(t_{j}\right)=f_{j} \vec{r}\left(t_{0}\right)+g_{j} \vec{v}\left(t_{0}\right) \\
\vec{v}\left(t_{j}\right)=\dot{f}_{j} \vec{r}\left(t_{0}\right)+\dot{g}_{j} \vec{v}\left(t_{0}\right)
\end{array}\right.
$$

where $\dot{f}=\frac{d}{d \tau} f, \dot{g}=\frac{d}{d \tau} g$ and $f_{j}$ and $g_{j}$ are power series in the time differences $\tau_{j}=t_{j}-t_{0}$,

$$
\left\{\begin{array}{l}
f_{j}=1-\frac{1}{2} \frac{\tau_{j}^{2}}{r^{3}\left(t_{0}\right)}+O\left(\tau_{j}^{3}\right) \\
g_{j}=\tau-\frac{1}{6} \frac{\tau_{j}^{3}}{r^{3}\left(t_{0}\right)}+O\left(\tau_{j}^{4}\right)
\end{array}\right.
$$

From equations (6) we can find the following expressions for $r\left(t_{j}\right), v\left(t_{j}\right), \vartheta_{r}\left(t_{j}\right)$, $\vartheta_{v}\left(t_{j}\right)$ and $\alpha\left(t_{j}\right), j=1, \ldots, N$, in terms of the initial conditions:

$$
\begin{cases}r^{2}\left(t_{j}\right)= & f_{j}^{2} r^{2}\left(t_{0}\right)+g_{j}^{2} v^{2}\left(t_{0}\right)+2 f_{j} g_{j} r\left(t_{0}\right) v\left(t_{0}\right)\left(H_{0}+K_{0}\right) \\ v^{2}\left(t_{j}\right)= & \dot{f}_{j}^{2} r^{2}\left(t_{0}\right)+\dot{g}_{j}{ }^{2} v^{2}\left(t_{0}\right)+2 \dot{f}_{j} \dot{g}_{j} r\left(t_{0}\right) v\left(t_{0}\right)\left(H_{0}+K_{0}\right) \\ \cos \left(\vartheta_{r}\left(t_{j}\right)\right)= & \frac{1}{r\left(t_{j}\right)}\left(f_{j} r\left(t_{0}\right) \cos \left(\vartheta_{r}\left(t_{0}\right)\right)+g_{j} v\left(t_{0}\right) \cos \left(\vartheta_{v}\left(t_{0}\right)\right)\right) \\ \cos \left(\vartheta_{v}\left(t_{j}\right)\right)= & \frac{1}{v\left(t_{j}\right)}\left(\dot{f}_{j} r\left(t_{0}\right) \cos \left(\vartheta_{r}\left(t_{0}\right)\right)+\dot{g}_{j} v\left(t_{0}\right) \cos \left(\vartheta_{v}\left(t_{0}\right)\right)\right) \\ \cos \left(\alpha\left(t_{j}\right)\right)= & \frac{\cos \left(\alpha\left(t_{0}\right)\right)}{r\left(t_{0}\right) v\left(t_{0}\right) H_{0}}\left[f_{j} \dot{f}_{j} r^{2}\left(t_{0}\right) \sin ^{2}\left(\vartheta_{r}\left(t_{0}\right)\right)+\right. \\ & \left.+g_{j} \dot{g}_{j} v^{2}\left(t_{0}\right) \sin ^{2}\left(\vartheta_{v}\left(t_{0}\right)\right)+\left(\frac{d}{d \tau}\left(f_{j} g_{j}\right)\right) r\left(t_{j}\right) v\left(t_{j}\right) H_{0}\right]\end{cases}
$$

with $H_{0}=\left(\sin \left(\vartheta_{r}\left(t_{0}\right)\right) \sin \left(\vartheta_{v}\left(t_{0}\right)\right) \cos \left(\alpha\left(t_{0}\right)\right)\right)$ and $K_{0}=\left(\cos \left(\vartheta_{r}\left(t_{0}\right)\right) \cos \left(\vartheta_{v}\left(t_{0}\right)\right)\right)$. From these equations it is then possible to obtain a system of $2(N+1)$ equations, 
the observations, in the initial conditions. The system is given by

$$
\left\{\begin{aligned}
F_{j}^{1}= & {\left[R^{2}+f_{j}^{2} r^{2}\left(t_{0}\right)+g_{j}^{2} v^{2}\left(t_{0}\right)+2 f_{j} g_{j} r\left(t_{0}\right) v\left(t_{0}\right)\left(H_{0}+K_{0}\right)+\right.} \\
& \left.2 R\left(f_{j} r\left(t_{0}\right) \cos \left(\vartheta_{r}\left(t_{0}\right)\right)+g_{j} v\left(t_{0}\right) \cos \left(\vartheta_{v}\left(t_{0}\right)\right)\right)\right]^{\frac{1}{2}} \\
F_{j}^{1} F_{j}^{2}= & f_{j} \dot{f}_{j} r^{2}\left(t_{0}\right)+g_{j} \dot{g}_{j} v^{2}\left(t_{0}\right)+\left(\frac{d}{d \tau}\left(f_{j} g_{j}\right)\right) r\left(t_{0}\right) v\left(t_{0}\right)\left(H_{0}+K_{0}\right)+ \\
& R\left(\dot{f}_{j} r\left(t_{0}\right) \cos \left(\vartheta_{r}\left(t_{0}\right)\right)+\dot{g}_{j} v\left(t_{0}\right) \cos \left(\vartheta_{v}\left(t_{0}\right)\right)\right)
\end{aligned}\right.
$$

for $j=1, \ldots, N$, and equations (5) for $j=0$. In these equations $\beta\left(t_{0}\right)$ does not appear, thus $\frac{\partial}{\partial \beta\left(t_{0}\right)} F_{j}^{i}=0$ for each $i=1,2$ and $j=0, \ldots, N$. Hence for all $g \in S^{1}$, we have that $F(g \vec{p})=F(\vec{p})$, because, as we remarked before, $S^{1}$ affects only $\beta\left(t_{0}\right)$.

We have thus found a one dimensional group of symmetry for the observations function $F: P \rightarrow O$. This implies that it is impossible to determine one of the six parameters that give the orbit of the satellite. We ask now whether it is possible to find other symmetries in the problem.

For $N+1=3$, we obtain for the initial conditions a system of six equations where only five of the initial conditions appear. Generally, we would then expect to find a unique solution to the system. This would show that $S^{1}$ is the only group of symmetry of the problem. But it would be cumbersome to prove that our system of equations is generic, so we restrict ourselves to prove the uniqueness of the symmetry group $S^{1}$ if we assume that the set of observations is large enough and well distributed in time. Some particular geometrical configurations are proved to be more degenerate than all the others (see also (Wood, 1986)): the cases of the satellite planetocentric orbit in the plane $x z$ orthogonal to the line of sight (i.e. the Earth-planet vector $\vec{R}$ ) or in the planes that contain $\vec{R}$ (see Figure 1).

Theorem 3.2. Excluding the particular geometrical configurations listed above, the only connected group of symmetries applicable to an arbitrary set of observations for the orbit determination of a satellite around another planet, with no relative motion between the Earth and the planet, and the planet spherically symmetric, is given by the rotations group $S^{1}$.

Proof. If the symmetry holds whatever the set of observations, then we can assume to know exactly the observations function $F(t)$ for any time $t$.

Let's consider the keplerian elements of the planetocentric orbit of the satellite with respect to the plane orthogonal to the line of sight, that is the plane orthogonal to the vector $\vec{R}$ between the Earth and the planet. From Theorem 3.1, we have that it is impossible to determine the longitude of the ascending node $\Omega$ of the satellite's orbit. We now show that, with our hypotheses it is possible to determine all the other orbital elements, if we are not in the geometrically degenerate cases listed above, that correspond to inclination $i=0$ or $i= \pm \frac{\pi}{2}$. 
First of all we notice that to know $F(t)$ for any $t$ implies that we know the period $T$ of the satellite's orbit. Then, by Kepler third law, we know the semi-major axis $a$ of the orbit ${ }^{\star}$.

Consider first the case with eccentricity $e \neq 0$. Then we use the expansions of the cartesian coordinates relative to the orbital plane in Fourier series on the mean anomaly $l$ (Brouwer and Clemence, 1961). If we denote by $f$ the true anomaly and by $r$ the radius, we have

$$
\left\{\begin{array}{l}
r \cos f=-\frac{3}{2} a e+a \cos l+\frac{1}{2} a e \cos 2 l+O\left(e^{2}\right) \\
r \sin f=a \sin l+\frac{1}{2} a e \sin 2 l+O\left(e^{2}\right)
\end{array}\right.
$$

We can express the observations function in terms of $r \cos f$ and $r \sin f$, obtaining the Fourier series of $\left(F^{1}(t)\right)^{2}$ and $F^{1}(t) F^{2}(t)$ on the mean anomaly $l$. We use only the approximations to the first order in the eccentricity. From these series, we can obtain the coefficients and equate them to the coefficients explicitly computed from the analytical expressions of the observations function. We write

$$
\left\{\begin{array}{l}
\left(F^{1}(t)\right)^{2}=f_{1}+f_{2} \cos l+f_{3} \sin l+\ldots \\
F^{1}(t) F^{2}(t)=f_{4}+f_{5} \sin l+f_{6} \cos l+\ldots
\end{array}\right.
$$

where $f_{j}=f_{j}(e, \omega, i)$, with $\omega$ the argument of the pericenter and $i$ the inclination, and the dots stand for the higher frequency Fourier terms. Thus, we obtain six equations on the variables $e, \omega, i$ with a unique solution. Indeed if we consider systems of three equations, we show that it is possible to build a non-degenerate system. Consider the system $\left(f_{1}, f_{3}, f_{5}\right)$. The Jacobian $J$ of the system is

$$
J=-6 a^{3} R^{2} e \sin i \cos i,
$$

hence if $e \neq 0$, then $J \neq 0$ and the system gives a unique solution for $e, \omega, i$ (assuming that $\sin i$ and $\cos i$ are $\neq 0$ ).

It remains to determine the time $\tau$ of passage from the perihelion. Once the elemente $(a, e, i, \omega)$ are known and the value for $R$ is given ${ }^{\star}$, we know $\|\vec{r}(\tau)\|=$ $a(1-e)$, and the value for $\|\vec{r}(\tau)+\vec{R}\|=F^{1}(\tau)$ can be obtained. Comparing this value with the observations, and using the fact that the sectorial velocity is known from the angular momentum, it is possible to find $\tau$.

In the case $e=0$, we only have to determine the inclination $i$. Looking at the power series for $\left(F^{1}(t)\right)^{2}$, we find

$$
\left\{\begin{array}{l}
f_{1}=a^{2}+R^{2} \\
f_{2}=0 \\
f_{3}=2 a R \sin i
\end{array}\right.
$$

* We assume the mass of the planet as known, although it could also be determined from $F(t)$.

* We assume the distance Earth-planet to be known although it could also be determined from $F(t)$ (Milani et al., 2001a). 
where we assume to measure the mean anomaly $l$ from the ascending node. Note that in this case the mean anomaly and the true anomaly are the same. Thus knowing the numerical value for $f_{3}$, we find the inclination $i$. In this case to find the time $\tau$ of passage from the ascending node, it is enough to look at the observations for $F^{1}(t)$ and select $\tau$ such that $F^{1}(\tau)=\frac{1}{2}\left(\max F^{1}(t)+\min F^{1}(t)\right)$, and $\frac{d}{d t} F^{1}(\tau)>0$ or $<0$, according to the sign of the inclination.

We have thus obtained all the keplerian elements of the satellite's planetocentric orbit but the longitude of the ascending node $\Omega$. Then by Theorem 2.3 the dimension of the symmetry group is 1 . We already have, by Theorem 3.1, a connected Lie group of dimension 1 , thus the connected component of the identity of the symmetry group must coincide with the rotations group of Theorem 3.1.

\section{The approximated symmetries}

We study the situation in the two different cases in which either our system is perturbed by a relative motion between the Earth and the planet or the planet is slightly oblate. We begin with the study of the former case.

\subsection{Relative motion between the Earth and the planet}

We still consider the two body problem as our model for the orbit of the satellite around the spheric planet, but we suppose that our system of coordinates $x y z$ is slowly rotating. Since the choice of the reference plane $x y$ is arbitrary, we can assume that the rotation takes place around the $z$ axis.

We have a finite set $t_{j}$ with $j=0, \ldots, N$ of times of the observations. Let $t_{0}$ be our reference epoch, so the quantities with subscript 0 are considered as initial conditions. Let's call $x^{j} y^{j} z^{j}$ the system of coordinates at the time $t_{j}$, with the $y^{j}$ axis in direction of the planet. We call $\varepsilon_{j}$ the angle between the axis $y^{j}$ and $y^{0}$, and suppose that $\varepsilon_{j}=k_{j} \varepsilon$ for each $j$, with $\varepsilon<<1$ and $\left|k_{j}\right| \leq 1$. Then the matrix of coordinates changing between $x^{j} y^{j} z^{j}$ and $x^{0} y^{0} z^{0}$ is given by

$$
A=\left(\begin{array}{ccc}
\cos \varepsilon_{j} & \sin \varepsilon_{j} & 0 \\
-\sin \varepsilon_{j} & \cos \varepsilon_{j} & 0 \\
0 & 0 & 1
\end{array}\right)
$$

Finally let's call $\left(r\left(t_{j}\right), \vartheta_{r}\left(t_{j}\right), \varphi_{r}\left(t_{j}\right)\right)$ and $\left(v\left(t_{j}\right), \vartheta_{v}\left(t_{j}\right), \varphi_{v}\left(t_{j}\right)\right)$ the coordinates of the radius vector planet-satellite and of the satellite's planetocentric velocity at time $t_{j}$. The components of the observations function $F$ are then given by

$$
\left\{\begin{array}{l}
F_{j}^{1}=\left\|\vec{r}\left(t_{j}\right)+\vec{R}\left(t_{j}\right)\right\| \\
F_{j}^{2}=\frac{d}{d t}\left\|\vec{r}\left(t_{j}\right)+\vec{R}\left(t_{j}\right)\right\|
\end{array} \quad j=0, \ldots, N .\right.
$$


Theorem 4.1. The normal matrix $C$ of our system has a column and a row of elements of the order $O(\varepsilon)$ and an element of the diagonal of the order $O\left(\varepsilon^{2}\right)$.

Proof. Let us consider one of the observations, assuming that the observation time $t_{j}$ is not at the epoch $t_{0}$.

From the relative motion of the Earth and the planet, we have that their reciprocal distance slightly changes, so we can write $R\left(t_{j}\right)=R\left(t_{0}\right)+\eta_{j}$, but this change doesn't affect at all our computation. So we can suppose $R_{j}=R_{0}=R$.

As in the proof of Theorem 3.1, we find a relation between the components of the vectors at time $t_{j}$ and those at the epoch $t_{0}$. To do that, we use the expression of the vectors at time $t_{j}$ in the coordinates system $x^{0} y^{0} z^{0}$. Then we use the following notation: $x^{i}\left(t_{j}\right) y^{i}\left(t_{j}\right) z^{i}\left(t_{j}\right)$ denote the rectangular coordinates of a vector relative to time $t_{j}$ in the reference system at time $t_{i}$. A subscript $r$ or $v$ indicates whether we are considering the coordinates of the radius vector or of the velocity vector. The same notation is used for the coordinates $\left(r, \vartheta_{r}, \varphi_{r}\right)$ and $\left(v, \vartheta_{v}, \varphi_{v}\right)$.

Using the matrix $A$ of transformations, we get

$$
\left\{\begin{aligned}
x_{r}^{j}\left(t_{j}\right) & =r\left(t_{j}\right) \sin \left(\vartheta_{r}^{j}\left(t_{j}\right)\right) \cos \left(\varphi_{r}^{j}\left(t_{j}\right)\right)= \\
& =r\left(t_{j}\right)\left(\cos \varepsilon_{j} \sin \left(\vartheta_{r}^{0}\left(t_{j}\right)\right) \cos \left(\varphi_{r}^{0}\left(t_{j}\right)\right)+\sin \varepsilon_{j} \cos \left(\vartheta_{r}^{0}\left(t_{j}\right)\right)\right. \\
y_{r}^{j}\left(t_{j}\right) & =r\left(t_{j}\right) \cos \left(\vartheta_{r}^{j}\left(t_{j}\right)\right)=r\left(t_{j}\right)\left(-\sin \varepsilon_{j} \sin \left(\vartheta_{r}^{0}\left(t_{j}\right)\right) \cos \left(\varphi_{r}^{0}\left(t_{j}\right)\right)\right. \\
& \left.+\cos \varepsilon_{j} \cos \left(\vartheta_{r}^{0}\left(t_{j}\right)\right)\right) \\
z_{r}^{j}\left(t_{j}\right) & =r\left(t_{j}\right) \sin \left(\vartheta_{r}^{j}\left(t_{j}\right)\right) \sin \left(\varphi_{r}^{j}\left(t_{j}\right)\right)=r\left(t_{j}\right) \sin \left(\vartheta_{r}^{0}\left(t_{j}\right) \sin \left(\varphi_{r}^{0}\left(t_{j}\right)\right)\right.
\end{aligned}\right.
$$

and similarly for $v$.

From the equations (10), we can get $\varphi_{r}^{j}\left(t_{j}\right)$ and $\vartheta_{r}^{j}\left(t_{j}\right)$ as functions of the components of $\vec{r}^{0}\left(t_{j}\right)$. We have

$$
\left\{\begin{aligned}
\varphi_{r}^{j}\left(t_{j}\right)= & \arctan \left(\frac{\sin \left(\vartheta_{r}^{0}\left(t_{j}\right)\right) \sin \left(\varphi_{r}^{0}\left(t_{j}\right)\right)}{\sin \left(\vartheta_{r}^{0}\left(t_{j}\right)\right) \cos \left(\varphi_{r}^{0}\left(t_{j}\right)\right) \cos \varepsilon_{j}+\cos \left(\vartheta_{r}^{j}\left(t_{j}\right)\right) \sin \varepsilon_{j}}\right)= \\
= & \varphi_{r}^{0}\left(t_{j}\right)+O(\varepsilon) \\
\sin \left(\vartheta_{r}^{j}\left(t_{j}\right)\right)= & \pm\left(1-\sin ^{2}\left(\vartheta_{r}^{0}\left(t_{j}\right)\right) \cos ^{2}\left(\varphi_{r}^{0}\left(t_{j}\right)\right) \sin ^{2} \varepsilon_{j}-\cos ^{2}\left(\vartheta_{r}^{0}\left(t_{j}\right)\right)\right. \\
& \left.\cos ^{2} \varepsilon_{j}+\frac{1}{2} \sin \left(2 \vartheta_{r}^{0}\left(t_{j}\right)\right) \cos \left(\varphi_{r}^{0}\left(t_{j}\right)\right) \sin 2 \varepsilon_{j}\right)^{\frac{1}{2}} \\
= & \sin \left(\vartheta_{r}^{0}\left(t_{j}\right)\right)+O(\varepsilon) \\
\cos \left(\vartheta_{r}^{j}\left(t_{j}\right)\right)= & \cos \left(\vartheta_{r}^{0}\left(t_{j}\right)\right) \cos \varepsilon_{j}-\sin \left(\vartheta_{r}^{0}\left(t_{j}\right)\right) \cos \left(\varphi_{r}^{0}\left(t_{j}\right)\right) \sin \varepsilon_{j}= \\
= & \cos \left(\vartheta_{r}^{0}\left(t_{j}\right)\right)+O(\varepsilon)
\end{aligned}\right.
$$


where we can choose the right signs, considering the fact that, being $\varepsilon$ small, $\varphi_{r}^{j}\left(t_{j}\right)$ and $\vartheta_{r}^{j}\left(t_{j}\right)$ are small perturbations of $\varphi_{r}^{0}\left(t_{j}\right)$ and $\vartheta_{r}^{0}\left(t_{j}\right)$. Similar relations can be found for the velocity components.

Using equations (5) and (11), we can write the observations function $F$ as a function of the variables $R, r\left(t_{j}\right), v\left(t_{j}\right), \varphi_{r}^{0}\left(t_{j}\right), \vartheta_{r}^{0}\left(t_{j}\right), \varphi_{v}^{0}\left(t_{j}\right)$ and $\vartheta_{v}^{0}\left(t_{j}\right)$. Let then $\alpha^{0}\left(t_{j}\right)=\varphi_{r}^{0}\left(t_{j}\right)-\varphi_{v}^{0}\left(t_{j}\right)$ and $\beta^{0}\left(t_{j}\right)=\varphi_{r}^{0}\left(t_{j}\right)+\varphi_{v}^{0}\left(t_{j}\right)$. If we want to find the dependence of the normal matrix $C$ on the small parameter $\varepsilon$, we have to consider the derivatives of $F_{j}^{i}, i=1,2$ with respect to $\beta\left(t_{0}\right)$.

Being $\beta^{0}\left(t_{j}\right)$ a small perturbation of $\beta\left(t_{0}\right)$, we can suppose that $\beta^{0}\left(t_{j}\right)=\beta\left(t_{0}\right)+$ $O(\varepsilon)$, so that $\frac{\partial}{\partial \beta\left(t_{0}\right)} \beta^{0}\left(t_{j}\right)=1+O(\varepsilon)$. Then we find that

$$
\frac{\partial}{\partial \beta\left(t_{0}\right)} F_{j}^{i}=(1+O(\varepsilon)) \frac{\partial}{\partial \beta^{0}\left(t_{j}\right)} F_{j}^{i}, \quad i=1,2 .
$$

Using equations (11), the zero order in $\varepsilon$ of the derivatives $\frac{\partial}{\partial \beta^{0}\left(t_{j}\right)} F_{j}^{i}, i=1,2$ is computed by equations (5) and (8), and is null as in Theorem 3.1, then

$$
\left\{\begin{array}{l}
\frac{\partial}{\partial \beta\left(t_{0}\right)} F_{j}^{1}=O(\varepsilon) \\
\frac{\partial}{\partial \beta\left(t_{0}\right)} F_{j}^{2}=O(\varepsilon)
\end{array}\right.
$$

From equations (12) and the particular form of the normal matrix $C$, it follows that $C$ has a column and a row with elements of the order $O(\varepsilon)$, and a diagonal element of order $O\left(\varepsilon^{2}\right)$.

\subsection{Oblateness of the planet}

We study now the case in which the planet is no more spherically symmetric, but we introduce a small oblateness of the planet, measured by the constant $J_{2}$. In this case, we suppose to have again no relative motion between the Earth and the planet. We consider only a fixed coordinates system $x y z$, as described in Section 3.1.

The equations we consider to model the motion of the satellite around the planet are those of the perturbed two body problem, where the potential is now given by the sum of the general term $U_{0}$ and the perturbative term $U_{1}$ (Roy, 1978). We have

$$
\left\{\begin{array}{l}
U_{0}=\frac{\mu}{r} \\
U_{1}=-\frac{\mu}{r} J_{2}\left(\frac{A}{r}\right)^{2} P_{2}(\sin \delta)
\end{array}\right.
$$

where $r$ is the distance planet-satellite, $A$ is the equatorial radius of the planet, $\mu$ is the gravitational term, $P_{2}(x)$ is the second Legendre polynomial and $\delta$ is the latitude of the satellite with respect to the equator of the planet.

We call $t_{j}$ with $j=0, \ldots, N$ the times of the observations, and suppose that $t_{j}-t_{0}=k_{j} \Delta t$ for each $j=1, \ldots, N$, with $\left|k_{j}\right|<1$. Again $\left(r\left(t_{j}\right), \vartheta_{r}\left(t_{j}\right), \varphi_{r}\left(t_{j}\right)\right)$ and $\left(v\left(t_{j}\right), \vartheta_{v}\left(t_{j}\right), \varphi_{v}\left(t_{j}\right)\right)$ are the coordinates of the satellite at time $t_{j}$. 
Theorem 4.2. Let's define $\eta=J_{2} \Delta t$ and suppose $\eta<<1$ and $J_{2}<<1$, if we define $\varepsilon=\max \left(J_{2}, \eta\right)$ then the normal matrix of the observations has one column and one row with elements of the order $O(\varepsilon)$, and one element on the diagonal of order $O\left(\varepsilon^{2}\right)$.

Proof. As in Theorem 4.1, let us consider one observation at time $t_{j}$ different from the epoch $t_{0}$.

As in the proof of Theorem 4.1, we know that it is enough to prove that the components $F_{j}^{i}, i=1,2$ of the observations function have their derivatives with respect to $\beta\left(t_{0}\right)=\varphi_{r}\left(t_{0}\right)+\varphi_{v}\left(t_{0}\right)$ of the order $O(\varepsilon)$. In this case we'll use the relations between our coordinates $\left(r\left(t_{j}\right), \vartheta_{r}\left(t_{j}\right), \varphi_{r}\left(t_{j}\right)\right)$ and $\left(v\left(t_{j}\right), \vartheta_{v}\left(t_{j}\right), \varphi_{v}\left(t_{j}\right)\right)$ and the keplerian orbital elements of the satellite, i.e. $a, e, i, \omega, \Omega, f$. So now we can use the perturbations of the keplerian elements given by the perturbative term $U_{1}$ of the potential. If we write $U_{1}$ as function of the orbital elements, we get (Roy, 1978)

$$
U_{1}=\mu\left\{\frac{3}{2} \frac{J_{2} A^{2}}{a^{3}}\left(\frac{a}{r}\right)^{3}\left[\frac{1}{3}-\frac{1}{2} \sin ^{2} i+\frac{1}{2} \sin ^{2} i \cos 2(f+\omega)\right]\right\} .
$$

The secular part of $U_{1}$, called $\bar{U}_{1}$ is given by

$$
\bar{U}_{1}=\frac{3}{2} \frac{\mu J_{2} A^{2}}{a^{3}}\left(\frac{1}{3}-\frac{1}{2} \sin ^{2} i\right)\left(1-e^{2}\right)^{-\frac{3}{2}}
$$

and the short periodic perturbations are of order $O\left(J_{2}\right)$.

From the usual equations for the perturbations of the keplerian elements of an elliptical orbit, due to the secular perturbative potential (Roy, 1978), and from equation (15), we can derive the orbital elements at time $t_{j}$, that is after $k_{j} \Delta t$ from $t_{0}$, our reference epoch. We have that the semi-major axis $a$, the eccentricity $e$ and the inclination $i$ change only for short periodic perturbations, thus by $O\left(J_{2}\right)$, while for the other elements we have

$$
\left\{\begin{aligned}
\omega=\omega_{0}+\Delta \omega= & \omega_{0}+\left[\frac{3}{2} \frac{A^{2} n_{0}}{p^{2}}\left(2-\frac{5}{2} \sin ^{2} i\right)\right] \eta+O\left(\eta J_{2}\right)+O\left(J_{2}\right) \\
\Omega=\Omega_{0}+\Delta \Omega= & \Omega_{0}-\left[\frac{3}{2} \frac{A^{2} n_{0}}{p^{2}}(\cos i)\right] \eta+O\left(\eta J_{2}\right)+O\left(J_{2}\right) \\
l=l_{0}+\Delta l= & l_{0}+\left[\frac{3}{2} \frac{A^{2} n_{0}}{p^{2}}\left(1-\frac{3}{2} \sin ^{2} i\right)\left(1-e^{2}\right)^{\frac{1}{2}}\right] \eta+ \\
& +O\left(\eta J_{2}\right)+O\left(J_{2}\right)
\end{aligned}\right.
$$

where the subscript 0 refers to the unperturbed elements, and $n_{0}$ is the mean motion. In equations (16) we have used the mean anomaly $l$, instead of the true anomaly $f$, but the two are related by

$$
\frac{d f}{d l}=\frac{a^{2}}{r^{2}} \sqrt{1-e^{2}}
$$


Clearly the components of the radius vector and of the velocity at time $t_{0}$ depend only on the unperturbed orbital elements, whereas the components of the quantities at time $t_{j}$ depend on the perturbations of the orbital elements, so on $\varepsilon$. Let's call for simplicity of notation, $\overrightarrow{o r} b$ the vector of the orbital elements, then we can write

$$
\left\{\begin{array}{c}
r\left(t_{j}\right)=r\left(\overrightarrow{o r} b_{0}\right)+O(\varepsilon)=r\left(t_{0}\right)+O(\varepsilon) \\
\vartheta_{r}\left(t_{j}\right)=\vartheta_{r}\left(\overrightarrow{o r} b_{0}\right)+O(\varepsilon)=\vartheta_{r}\left(t_{0}\right)+O(\varepsilon) \\
\varphi_{r}\left(t_{j}\right)=\varphi_{r}\left(\overrightarrow{o r} b_{0}\right)+O(\varepsilon)=\varphi_{r}\left(t_{0}\right)+O(\varepsilon)
\end{array}\right.
$$

Using the well-known transformations from the keplerian orbital elements to the cartesian coordinates, and then to our coordinates, we can write $F_{j}^{i}, i=1,2$ as functions of $\left(r\left(t_{0}\right), \vartheta_{r}\left(t_{0}\right), \varphi_{r}\left(t_{0}\right)\right)$ and of $\left(v\left(t_{0}\right), \vartheta_{v}\left(t_{0}\right), \varphi_{v}\left(t_{0}\right)\right)$. Then we use $\alpha\left(t_{0}\right)=$ $\varphi_{r}\left(t_{0}\right)-\varphi_{v}\left(t_{0}\right)$ and $\beta\left(t_{0}\right)=\varphi_{r}\left(t_{0}\right)+\varphi_{v}\left(t_{0}\right)$, and we can derive our observations function with respect to $\beta\left(t_{0}\right)$. As in Theorem 4.1, we have $\frac{\partial}{\partial \beta\left(t_{0}\right)} F_{j}^{i}=O(\varepsilon), i=1,2$, then the theorem follows as before, and one of the elements on the diagonal of the normal matrix $C$ will be $\sum_{j=1}^{N}\left(\frac{\partial F_{j}^{1}}{\partial \beta\left(t_{0}\right)}\right)^{2}+\left(\frac{\partial F_{j}^{2}}{\partial \beta\left(t_{0}\right)}\right)^{2}=O\left(\varepsilon^{2}\right)$.

It is possible to obtain an estimate for the coefficients of the diagonal terms of order $O\left(\varepsilon^{2}\right)$ of the normal matrix $C$, due to the relative motion between Earth and the planet and in the case of oblateness of the planet. But if we are interested in the particular case of a satellite moving around a planet as Mercury, whose synodic period with respect to the Earth is much less than the period of the secular perturbations on the keplerian elements (see equations (16)), then it is clear that the more important term must be the one due to the relative motion of the Earth and the planet.

\section{The eigenvalues of the normal matrix}

We now try to quantify the effect of the perturbations, considered in the previous section, on the ideal problem with the exact symmetry. We have shown in Theorem 4.1 and 4.2 that the perturbations lead to a change in the normal matrix $C$. Namely the zero column and row are destroyed and there are a column and a row with elements of order $\varepsilon$ and a diagonal element of order $\varepsilon^{2}$. From the continuous dependence of the eigenvalues of a matrix from its elements, it follows that the normal matrix $C$ must have an eigenvalue $\lambda_{\varepsilon}$ close to 0 . It becomes important to find the leading terms of the expansions of $\lambda_{\varepsilon}$ in power series of $\varepsilon$.

We give here a brief presentation of the theory that allows us to find the order of magnitude of the eigenvalue $\lambda_{\varepsilon}$.

Let $A$ be a $6 \times 6$ matrix of the form

$$
A=\left(\begin{array}{cc}
0_{1 \times 1} & 0_{1 \times 5} \\
0_{5 \times 1} & B_{5 \times 5}
\end{array}\right)
$$


where $0_{i \times j}$ is a matrix of zeros of dimension $i \times j$, and $B$ is a $5 \times 5$ matrix with $\operatorname{det} B \neq 0$.

Let $\delta A$ be a small perturbation of the matrix $A$ of the form

$$
\delta A=\left(\begin{array}{ll}
\varepsilon_{1 \times 1}^{2} & \varepsilon_{1 \times 5} \\
\varepsilon_{5 \times 1} & \varepsilon_{5 \times 5}
\end{array}\right)
$$

with the same notation as in $A$, and the matrices denoted by $\varepsilon_{i \times j}$ and $\varepsilon_{1 \times 1}^{2}$ are made of elements of order $\varepsilon$ and $\varepsilon^{2}$, respectively.

From the form of the matrix $A$ we can see that $\operatorname{det} A=0$, and it has exactly one eigenvalue $\lambda=0$. Let's denote with $p(x)$ the characteristic polynomial of $A$ and with $q(x)$ the characteristic polynomial of $B$. We have, from equation (18), that $p(x)=x q(x)$ and that $p(0)=0$ and $q(0) \neq 0$.

Theorem 5.1. The matrix $A+\delta A$ has one eigenvalue of order $\varepsilon^{2}$.

Proof. Let $k(x)$ be the characteristic polynomial of the matrix $A+\delta A$. To prove the theorem we have to show that $k(x)$ has a zero of order $\varepsilon^{2}$.

First of all, we notice that $k(x)$ has the form $k(x)=x q(x)+\varepsilon^{2} r(x)$. Indeed, we know that $k(x)=\operatorname{det}[x I-(A+\delta A)]$, and to obtain $k(x)$ in the desired form we use the development of the determinant by the first row.

From the continuous dependence of the zeroes of a polynomial with respect to its coefficients, we know that there must be a root of $k(x)$ near to 0 for small $\varepsilon$. Let's call $\lambda_{\varepsilon}$ this root. It is then possible to apply the Implicit Function Theorem to the function $G\left(x, \varepsilon^{2}\right)=x q(x)+\varepsilon^{2} r(x)$. We have $G(0,0)=0$ and $G_{x}(0,0)=$ $q(0)=\operatorname{det} B \neq 0$, then by the Implicit Function Theorem there exists a function $\bar{x}\left(\varepsilon^{2}\right)$ defined in a neighbourhood $U$ of 0 , such that $G\left(\bar{x}\left(\varepsilon^{2}\right), \varepsilon^{2}\right)=0$ for all $\varepsilon^{2} \in U$. The function $\bar{x}\left(\varepsilon^{2}\right)$ defines the eigenvalue $\lambda_{\varepsilon}$ we are looking for. It holds

$$
\left.\frac{\partial \bar{x}\left(\varepsilon^{2}\right)}{\partial \varepsilon^{2}}\right|_{\varepsilon^{2}=0}=-\frac{G_{\varepsilon^{2}}(0,0)}{G_{x}(0,0)}=-\frac{r(0)}{q(0)},
$$

from which it follows

$$
\lambda_{\varepsilon}=-\varepsilon^{2} \frac{r(0)}{q(0)}+O\left(\varepsilon^{4}\right),
$$

where $q(0)=\operatorname{det} B$.

Corollary 5.2. The normal matrix $C$ of the observations function $F$ has an eigenvalue of order $\varepsilon^{2}$.

Proof. It easily follows from Theorem 5.1, noting that $C$ is of the form $A+\delta A$. Moreover, we proved in Theorem 3.1 that in the ideal case $C$ has only one eigenvalue $\lambda=0$, so that we can suppose that $\operatorname{det} C=x q(x)$ with $q(0) \neq 0$. So we are in the hypothesis of Theorem 5.1 and the corollary follows. 
We have thus proved that the approximated symmetries in our system cause the presence of one eigenvalue of the normal matrix $C$ of the order of the square of the perturbation. Then we can say that the normal matrix $C$ has an approximated rank deficiency of order 1 (see Definition 2.1).

Going back to the theory of orbit determination ((Milani and Melchioni, 1989) and Sect. 2), we see that an approximated rank deficiency of order 1 for the normal matrix leads, using the notion of confidence ellipsoid (eqn. (1)), to a loss of precision in the determination of the orbit. Indeed we find that one of the axes of the confidence ellipsoid (given by the inverse of the eigenvalue of order $\varepsilon^{2}$ ) is much bigger than the others axes. Hence the parameter that corresponds to this axis is determined much worse than the others.

\section{Controlling the degeneracy}

The conclusion, reached in the previous Section, is that in a problem of orbit determination around another planet one of the parameters to be determined from the observations cannot be found to belong to a small set of possible values, but has an uncertainty much bigger than the other parameters. Then, how should we proceed to prevent the orbit determination to be degraded by this effect?

Three different approaches can be used for this. The first one is mathematically elegant but not practical. It is possible to select a set of only five orbital elements, in such a way that the reduced $5 \times 5$ normal matrix does not have small eigenvalues. From the proofs of Theorems 3.1 and 3.2, a set of keplerian orbital elements $(a, e, i, \omega, \Omega, l)$ where the inclination and node $i, \Omega$ are with respect to the plane $\rho$, the plane through the center of the planet orthogonal to the Earth-planet direction, would have the desired property, with the longitude of the node $\Omega$ as the variable to be excluded, allowing to solve for $(a, e, i, \omega, l)$ without degeneracy. There are three drawbacks: (1) this set of elements is not convenient to use to describe the orbit, which is normally expressed in elements with the planetary equator as reference plane; (2) the reference plane $\rho$ changes, although slowly, and to connect two orbit determinations done at well separated times it is necessary to assume some value also for $\Omega$; (3) a complete set of orbital elements is anyway necessary for most applications, e.g., scheduling of remote sensing observations.

The second solution is the method of collocation, namely, some a priori value is assumed for all six initial conditions. Let the uncertainty of this a priori knowledge be expressed by the $6 \times 6$ covariance matrix $\Gamma_{0}$. Then the set of observations is augmented by adding six "a priori observations", in which the values of the initial conditions are "observed" to be equal to the a priori values with uncertainty expressed by a normal distribution with covariance $\Gamma_{0}$. This implies that the normal matrix $C$ is replaced by $C+C_{0}$ with $C_{0}=\Gamma_{0}^{-1}$ the "a priori normal matrix". If the matrix $\Gamma_{0}$ does not have too large eigenvalues, then $C_{0}$ does not have too small eigenvalues and therefore the approximate degeneracies are removed ((Arnold, 
1989), section 24). The question is where the a priori knowledge comes from, and the only reasonable answer is that it comes either from the propagation of an orbit determination performed at some previous time, or from an orbit determination done over a longer time span (and therefore much less affected by the approximate degeneracy). In both cases the real accuracy of the a priori knowledge (as opposed to the formal one) is degraded as result of dynamic model approximations, resulting in orbit propagation errors accumulating rapidly (e.g., quadratically) with time. Thus too tight assumptions on $\Gamma_{0}$ would give results with illusory accuracy. This method has been used in (Milani et al., 2001a) by assuming a priori uncertainties two orders of magnitude larger than the ones which can be obtained, for five out of six elements, in a short arc orbit determination.

The third approach is to constrain the solution in such a way that the rotations around the Earth-planet direction (with unit vector $\hat{R}$ ) are inhibited. This can be obtained by means of a single a priori observation of the quantity $\hat{R} \wedge \vec{r}$ with some arbitrary accuracy, corresponding to a normal distribution of errors with variance $\gamma_{0}$ (Milani et al., 1995). It is important to take into account that this a priori information (unlike the one of the previous method) is fictitious. Indeed it is convenient to assume that $\gamma_{0}$ is extremely small, thus removing any small eigenvalue from the augmented normal matrix. However, this implies that the initial conditions as determined have an illusory accuracy in the "weak" direction, and this results in the same problems of the first approach above. Thus the method of constraints can be useful when the orbit determination is not the primary goal, but an intermediate step towards the determination of other parameters of interest.

\section{Conclusions}

We have formulated a rigorous theory of rank deficiency due to symmetries in orbit determination problems, and analysed in particular the case of the orbit determination for a satellite of another planet, observed by range and range-rate tracking. Exact symmetry, thus exact degeneracy of the normal equations of the least square fit, occurs only if the planet motion is negligible, and the planet is modeled as spherically symmetric. In practice, the planet moves by a small angle on the celestial sphere during the time span of a tracking session, and the shape of the planet introduces small perturbations to the keplerian orbit of the satellite. In this realistic case the normal system is not anymore degenerate, but still it is poorly conditioned. This results in an increase of the uncertainty in the initial conditions determined on the basis of the tracking data: the amount of this increase can be estimated, for all planetocentric orbits, by the results of this paper.

In practice, the increase in the uncertainty is very significant for realistic planetary models and tracking schedules typical of planetary missions, thus this problem of approximated rank deficiency cannot be neglected in assessing the performance of a tracking system. There are solutions to this problem, as discussed in Section 
6, which allow to stabilize the solution and remove most of the negative effects, but the problem needs to be understood and taken into account in the design of the orbit determination algorithms, as in (Milani et al., 2001a), (Milani et al., 2001b).

\section{References}

V.I. Arnold: 1989, Mathematical Methods of Classical Mechanics, Springer-Verlag

D. Brouwer and G.M. Clemence: 1961, Methods of Celestial Mechanics, Academic Press, Inc.

P.R. Escobal: 1965, Methods of Orbit Determination, John Wiley \& Sons.

C.F. Gauss: 1809, Theoria motus corporum coelestium in sectionis conicis solem ambientum, Hamburg.

A. Milani, M. Carpino, A. Rossi, G. Catastini, S. Usai: 1995, "Local geodesy by satellite laser ranging: a European solution", Manuscripta Geodaetica, 20, 123-138

A. Milani and E. Melchioni: 1989, "Determination of a local geodetic network by multi-arc processing of a satellite laser ranges", In: F. Sansò F. and R. Rummel (eds) Theory of Satellite Geodesy and Gravity Field Determination, Springer-Verlag, pp 417-445

A. Milani, A. Rossi, D. Vokrouhlicky, D. Villani, C. Bonanno: 2001a, "Gravity field and rotation state of Mercury from the BepiColombo Radio Science Experiments", Planetary Space Science, 49, no. 14-15, 1579-1596

A. Milani, D. Vokrouhlicky, A. Rossi, D. Villani, C. Bonanno: 2001b, “Testing general relativity with the BepiColombo radio science experiment", submitted

A.E. Roy: 1978, Orbital Motion, Adam Hilger LTD.

R.K. Russell and S.W. Thurman: 1989, "An analytic development of orbit determination for a distant, planetary orbiter", Interplanetary Network Progress Report, electronic journal Jet Propulsion Laboratory, http://tmo.jpl.nasa.gov/tmo/progress_report/, 42-98, April-June 1989

L.J. Wood: 1986, "Orbit determination singularities in the Doppler tracking of a planetary system", J. Guidance and Control, 9, no. 4, 485-494 
symmetries.tex; 7/01/2002；14:45; p.18 